\title{
On the transient modelling of hydro-elastic interaction of gravity waves with an underwater elastic structure
}

\author{
N. M. Sudharsan* K. Murali ${ }^{\dagger}$
}

(Received 19 October 2004, revised 30 December 2005)

\begin{abstract}
Fluid structure interaction (FSI) of offshore structures is an important area of research. The behaviour of offshore structures in Numerical Wave Tanks possessing FsI capabilities is of significant industrial value. In FSI problems, it is necessary to interactively solve and analyse both the structural and fluid dynamics as a coupled system, because the structural response and fluid loading are mutually dependent. We present a methodology for analysing a two-dimensional fluid-structure interaction problem in the time domain. The methodology is based on a nonlinear velocity potential formulation and linear
\end{abstract}

*Dept. of Mechanical Engineering, College of Engineering, Guindy, Anna University, Chennai, InDIA, 600 025. mailto:sudharsanneasme.org

${ }^{\dagger}$ Dept. of Ocean Engineering, Indian Institute of Technology, Madras, Chennai IndiA, 600036.

See http://anziamj.austms.org.au/V46/CTAC2004/Sudharsan for this article, (C) Austral. Mathematical Soc. 2006. Published January 6, 2006. ISSN 1446-8735 
elastic theory. The transient forces exerted by the fluid on the structure vary due to the elastic response of the structure. The dynamics of the free surface and the structure are also analysed for selected scenarios. We demonstrates that it is important to develop similar tools capable of solving such FSI problems in order to perform reliable and complete analysis leading to better and safer design.

\section{Contents}

1 Introduction

C1354

2 Mathematical models and numerical strategies

C1356

2.1 Nonlinear free-surface flow . . . . . . . . . . . . . . C1356

2.2 Structural formulation . . . . . . . . . . . . . . . C C1358

2.3 Computational methods . . . . . . . . . . . . . . C1359

3 Results and discussion

C1360

3.1 Simulation scenarios . . . . . . . . . . . . . . C1361

3.2 Simulation results . . . . . . . . . . . . . . . . . C1362

4 Concluding remarks

C1368

References

C1369

\section{Introduction}

Transient behaviour of offshore structures is often nonlinear and involves coupled transient fluid-structure interaction (FSI). Research in this area is still at an early stage. Frequency domain models are linear and any nonlinearity emanating from either the fluid or structure behaviour will not be captured in the frequency domain. Laboratory based experiments have limitations, 
for example, scale effects and instrumentation difficulties, requiring one to resort to numerical simulations to study this class of problems. Here, it is not only necessary to consider the fluid forces due to the wave loading on the structure, but also the subsequent response of the structure to the applied load. Typically, the fluid dynamics is solved to obtain the forces on the structure. The structural dynamics is subsequently solved to obtain its response in terms of displacement to the applied load. However, if this displacement is large enough, it will significantly alter the boundary conditions of the fluid domain. This in turn will modify the force profile. It is therefore necessary to solve both the fluid and structure problems in a coupled manner to obtain the true behaviour of the structure and the corresponding fluid wave profile. As it is common for the fluid domain to be solved in an Eulerian reference frame and the structural domain in a Lagrangian framework, an Arbitrary Lagrangian-Eulerian (ALE) method is to be used.

The objective of the current work is to develop a fully nonlinear $3 \mathrm{D} \mathrm{Nu}-$ merical Wave Tank with FSI capabilities. This requires solving the nonlinear fluid and structure behaviour in the time domain. While full free-surface nonlinearity is considered for the waves, only geometric nonlinearity of the structure is accounted for. In this paper, a $2 \mathrm{D}$ formulation of the above problem is considered and reported. A velocity potential formulation is used for the fluid flow problem as the ocean wave flow field is mostly irrotational. The finite element method is chosen for solving this FSI problem. As the free surface needs to be captured at each time step, along with the new position of the structure, there is a need to choose a meshing scheme that will more aptly represent the wave surface profile. Therefore, unstructured triangular meshes are used to discretize the fluid and structural domain. Mesh smoothing is done at regular intervals to avoid skewed elements which lead to solution inaccuracy. 


\section{Mathematical models and numerical strategies}

The aim is to model both static and dynamic structural dynamics with FSI capabilities. The equations governing fluid flow are the Laplace equation with transient free surface and body boundary conditions. The free surface condition is nonlinear by nature. However, only a geometric nonlinearity exists on the body surface. Conversely, the equation governing the structure is based on the principle virtual work, and is considered both in dynamic and static form.

\subsection{Nonlinear free-surface flow}

The fluid is considered to be inviscid and incompressible and the flow irrotational. However, the free surface is considered fully time evolving and hence the governing free-surface condition becomes nonlinear. Under these conditions a velocity potential $\phi$ exists and the fluid flow is governed by the Laplace equation. The mathematical statement of the problem is

$$
\begin{aligned}
\nabla^{2} \phi & =0 \quad \text { in } \Omega \text { (fluid region), } \\
\frac{\partial \phi}{\partial n} & =f(\Gamma) \quad \text { on } \Gamma=S_{l}+S_{0}+S_{b}+S_{f} .
\end{aligned}
$$

Here $\Omega$ is the domain bounded by the surface $\Gamma$. The surface is subdivided into a body surface $S_{0}$, a free surface $S_{f}$, a sea-bed $S_{b}$ and two radiation boundaries $S_{l}$; all are assumed to be piece-wise continuous. The nonlinearity is introduced in the system through the full free-surface boundary conditions (on $S_{f}$ ) which written in Lagrangian form are

$$
\frac{d \phi}{d t}=g \eta-\frac{1}{2} \nabla \phi \cdot \nabla \phi,
$$




$$
\frac{d x}{d t}=\frac{\partial \phi}{\partial x}, \quad \frac{d y}{d t}=\frac{\partial \phi}{\partial y}
$$

where $g$ is the acceleration due to gravity and $\eta$ is the free surface elevation. Physically, equation (3) imposes a constant pressure on the free surface while equation (4) helps in keeping the free surface in phase with the water particles on it. Assuming a piston movement of the form $\delta=\delta_{0}(1-\cos \omega t)$, the wave making condition for the left-hand side boundary is

$$
\phi_{, x}=\omega \delta_{0} \sin \omega t .
$$

For the other surfaces $\left(S_{0}, S_{b}\right.$ and $\left.S_{l}\right)$ the condition of no flow across the boundary is used. These are Neumann type boundary conditions.

Equations (1)-(4) define a mixed Dirichlet-Neumann boundary value problem. The steady state equation (1) is repeatedly solved over time using transient boundary conditions (3)-(5). A finite element method is adapted to discretize equation (1). Details of the methodology may be found in $\mathrm{Wu}$ and Taylor [2].

Having obtained the quasi-steady velocity potentials, the transient pressure force on the structure due to the fluid flow is calculated using Bernoulli's equation. The instantaneous pressure force

$$
F=-\rho \frac{\partial}{\partial t} \int_{S_{0}} \phi n d s+\rho \int_{S_{0}}\left(\frac{\partial \phi}{\partial n} \nabla \phi-\frac{1}{2} \nabla \phi \nabla \phi\right) d s,
$$

where $\rho$ is the fluid density and $\mathbf{n}$ is the outward normal to the fluid or the inward normal to the structure. However, it is not straightforward to calculate the velocities (gradient of $\phi$ ) within the integrals as linear shape functions are used. Here again, the method proposed by $\mathrm{Wu}$ and Taylor [3] has been used. In order to minimise inaccuracies in this quasi-static procedure, the time step is kept small and an iterative loop in adopted in the FSI coupling. 


\subsection{Structural formulation}

The structure considered here is a submerged semi-infinite solid body that extends to infinity in the direction normal to the fluid flow. This reduces the structural problem to two dimensions and hence the deformation of the structure may be obtained by modelling it as a plane strain problem. The displacements of the structure are measured in the $x y$-coordinate system and are denoted by $\boldsymbol{U}(x, y)=(u, v)$ and $\boldsymbol{U}=\boldsymbol{U}^{S_{0}}$ on the surface $S_{0}$. The stresses and strains are linearly related through a material matrix. The basis of the displacement based finite element procedure is the principle of virtual displacements. It is stated that for any compatible small virtual displacements imposed on a body that is in a state of equilibrium, the total internal virtual work must equal to the total external virtual work:

$$
\int_{t} \int_{S} \varepsilon^{T} \sigma d A d t=\int_{t} \int_{S} \rho \dot{\boldsymbol{U}}^{T} \dot{\boldsymbol{U}} d A d t+\int_{t} \int_{\Gamma=S_{0}} \boldsymbol{U}^{S_{0} T} f_{S_{0}} d S d t .
$$

The discretized form of the governing equation of motion for the structural system without damping is

$$
[\mathbf{M}] \ddot{\boldsymbol{U}}+[\mathbf{K}] \boldsymbol{U}=[\mathbf{F}],
$$

where $[\mathbf{M}]$ and $[\mathbf{K}]$ are the structural mass and stiffness matrices, $\ddot{\boldsymbol{U}}$ and $\boldsymbol{U}$ are the generalized nodal accelerations and displacements, and $[\mathbf{F}]$ is the generalized force vector which is a summation of the external nodal forces $\left[\mathbf{F}_{\mathbf{e}}\right]$ and the nodal forces exerted on the structure due to the fluid oscillation $[\mathbf{P}]$, that is, $[\mathbf{F}]=\left[\mathbf{F}_{\mathbf{e}}\right]+[\mathbf{P}]$. The body is discretised with triangular finite elements. The fluid forces are obtained after each time step and are applied to the structure to obtain the static structural response. A dynamic structural analysis is also performed for certain cases. The methodology of solving the above system of matrices is given in [4]. A comparison of static and dynamic coupling and the need for added mass terms are discussed in detail in Section 3.2. 


\subsection{Computational methods}

The forgoing mathematical formulation looks simple. However, major computational difficulties lie in obtaining stable and accurate solutions over an extended period of time. This section summarises a sequential coupling procedure that has been successfully employed for obtaining a dynamic equilibrium of the hydrodynamic fluid-structure system considered here. The focus is on the 'conventional serial staggered' (Css) approach for dynamic coupling as presented by Farhat and Lesoinne [5]. In addition, as finite element procedures produce sparse matrices, the issues of node renumbering and mesh dependence of the solutions are also discussed.

The present sequential coupling procedure is initialised by solving the nonlinear free-surface problem. The fluid forces on the structure are then computed using equation (6) and imposed onto the structural nodes as per equation (7). The resulting structural displacements and velocities are obtained by solving the structural system. This yields the new body boundary position for the fluid domain along with the new velocity conditions. At the interface, no separation of fluid particles is allowed, that is the velocity of the fluid particle is equal to the structural displacement at that time interval $(\partial \phi / \partial x=\partial u / \partial t$ and $\partial \phi / \partial y=\partial v / \partial t)$. This results in an arbitrary displacement of the nodes along the structure boundary. Using these updated boundary conditions and the boundary position at the fluid-structure interface, solution of the fluid flow is obtained for the next time step. These steps are repeated iteratively until convergence, to march the fluid-structure dynamics in time. As the fluid node is not allowed to separate from the structure, continuity of normal velocity is satisfied implying a no-flow boundary condition. However, the tangential velocity is allowed as the structure itself undergoes deformation. Though there may be differences in the tangential velocity of fluid and structure at the interface, the iterative approach adopted is expected to reduce such inaccuracies. The mesh quality is verified at regular intervals for skewness and a Laplacian smoothing is performed when necessary. 
The Revere Cuthill-Mckee (RCM) algorithm has been used in the present study to reduce the bandwidth of the fluid system matrix. This ensures a compact storage of the matrix and speeds up the solution process enormously. A typical simulation where the domain is discretised with 4829 nodes the modified bandwidth has been 18 which is only $0.4 \%$ of the full matrix. A Laplacian smoothing algorithm [6] is used in order to maintain mesh quality resulting from dynamic mesh movement. This smoothing method was adopted after testing various mesh adaptation techniques for FSI problems involving dynamic boundaries [7]. The mesh quality (skewness) is finally tested by comparing the edge ratios of the triangular elements and Laplacian smoothing is performed until a good quality mesh is obtained. A singularity exists at the corner of the wave maker piston and the free surface. It is necessary to handle the singularity appropriately which otherwise would result in solution instabilities. In this work height of the corner node (the free surface position) is monitored. If the height of the corner node is above the wave maker piston then the calculated $\phi$ (free surface potential) is enforced. Otherwise a Neumann boundary condition of the known velocity of the wave maker piston is enforced.

In numerical computations, the accuracy of the solution depends on the mesh. It is a usual practice to define the mesh density in gravity wave simulations as the number of nodes per wavelength. Analysis shows that a mesh independent solution is reached for mesh densities $\left(N^{m}\right)$ above 40; details of the complete mesh independence analysis may be found in $[7,8]$. Validation is an essential requirement of any computational algorithm. The present fluid-structure algorithm has been extensively validated [8].

\section{Results and discussion}

The objective of the present study is to demonstrate a 'Numerical Wave Tank' with capabilities to perform hydro-elastic computations in addition 
to wave generation and absorption. The numerical experiments that follow are selected with this objective in mind. The discussions demonstrate the capabilities and usefulness of the present approach in simulating the hydroelastic behaviour of a typical underwater structure.

\subsection{Simulation scenarios}

The flow domain consists of a rectangular tank of length $60 d$, where $d$ is the depth of the undisturbed water; $d=1.0 \mathrm{~m}$ in all simulations. The domain is meshed with $N^{m}$ between 40 and 50. The structural configuration considered here is an underwater vertical wall of height $0.67 d$ and width $B=0.03125 d$. A total of nine combinations of simulation scenarios are considered by varying material properties, coupling method and wave frequency. The flexural rigidity of the structure has been varied by considering different values of Young's Modulus and Poisson ratio. The structure is placed at a distance of $6 d$ from the left boundary (the wave maker). The amplitude $\delta_{0}$ of the wave maker is maintained at $0.05 d$ for all the simulations considered for this study. Complete details of the various simulation scenarios considered are given in Table 1.

The range of material properties considered here covers a wide range of materials whose properties are available in standard tables. However, in order to further test the computations procedures used here, material properties outside the standard range also are used and they are reported as and when needed. For all computations the nondimensional time step $\Delta t^{*}=\Delta t \sqrt{g / d}=\pi / 120$ is used for both the fluid and structural solution. Note that the placement of structure close to the wave maker may cause re-reflection. The simulations could not be run for long durations without a total re-meshing of the computational domain due to mesh failure and the formation of skewed meshes. Placing the structure close to the wave maker ensures that waves reach the structure before mesh failure. As the focus of the present work is to study the nonlinear fluid structure coupling, the effects 
TABLE 1: Simulation Scenarios

\begin{tabular}{rlllll}
\hline Scenario & Structure & \multicolumn{2}{c}{ Material properties } & $\omega^{*}$ & Coupling \\
\hline (a) & Steel & $E=200 \mathrm{GPa}$ & $\mu=0.3$ & 1.0 & Dynamic \\
(b) & Arbitrary & $E=0.2 \mathrm{GPa}$ & $\mu=0.35$ & 1.0 & Dynamic \\
(c) & Arbitrary & $E=0.1 \mathrm{GPa}$ & $\mu=0.35$ & 1.0 & Dynamic \\
(d) & Steel & $E=200 \mathrm{GPa}$ & $\mu=0.3$ & 1.0 & Static \\
(e) & Arbitrary & $E=0.0025 \mathrm{GPa}$ & $\mu=0.35$ & 1.0 & Static \\
(f) & Steel & $E=200 \mathrm{GPa}$ & $\mu=0.3$ & 0.5 & Dynamic \\
(g) & HDPE & $E=0.8 \mathrm{GPa}$ & $\mu=0.35$ & 0.5 & Dynamic \\
(h) & Arbitrary & $E=0.2 \mathrm{GPa}$ & $\mu=0.35$ & 0.5 & Dynamic \\
(i) & Arbitrary & $E=0.1 \mathrm{GPa}$ & $\mu=0.35$ & 0.5 & Dynamic \\
\hline
\end{tabular}

of re-reflection of waves from the wave maker are ignored.

\subsection{Simulation results}

The transient pressure forces on the structure induced in the direction of wave propagation are shown in Figure 1 for different material properties of the structure. Periodicity of the force plots show the interactive behaviour of the fluid and the structure. The plots show that the structure starts to feel the fluid pressure approximately one cycle after initiation of the wave maker operation for wave frequency $\omega^{*}=1.0$. In contrast, for $\omega^{*}=0.5$, the structure starts to feel the wave force after approximately half a cycle. Observe in the figure that the forces on the structures are periodic and stable for the case of dynamic coupling, while static coupling over-predicts the forces, as also observed by Pal et al. [9]. Although the static and dynamic coupling initially respond with the same amplitude, the forces become unrealistic after some time when static coupling is employed. The simulations fail due to excessive mesh movement and formation of a negative element area. This indicates that the structure responds too rapidly and inclusion of mass and added-mass terms may give more realistic results. The time at which 

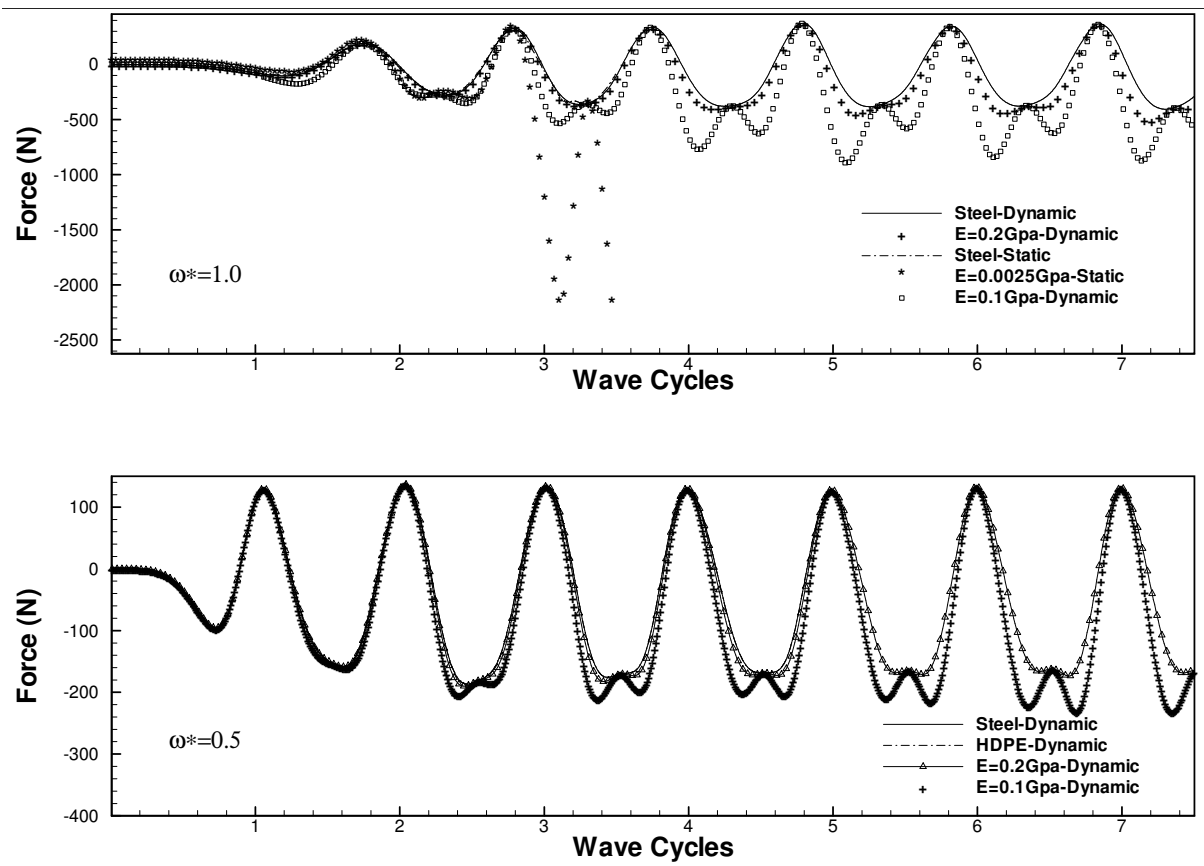

FiguRE 1: Transient wave forces on structures with different properties for $\omega^{*}=1.0$ and $\omega^{*}=0.5$. 
the structure becomes unrealistic varies for each simulation. For example, results for scenarios (a) and (d), representing dynamic and static coupling for steel, are virtually identical up to 3 cycles, after which the sudden increase in force causes mesh failure for static coupling. The same behaviour can also be observed for case (e). On inclusion of the full structural dynamics, the forces are controlled and stable. After some time a periodic force history is developed, indicating a stable range of the fluid-structure interaction process. This stable range develops after 3 cycles [1].

Different material properties lead to similar force patterns. However, there is a significant difference in the force magnitudes for different material characteristics. For $\omega^{*}=1.0$, when the elastic constant $E$ is reduced from $200 \mathrm{GPa}$ to $0.2 \mathrm{GPa}$, the force amplitude increases by about $30 \%$. This increase is about $100 \%$ when the $E$ is reduced from $0.2 \mathrm{GPa}$ to $0.1 \mathrm{GPa}$. This nonlinear relationship should be expected as the total system has geometric (due to dynamic interaction of the structure with the fluid) and mathematical nonlinearities (from the free surface). This indicates a significant need for transient coupled fluid-structure modelling for the present set of conditions.

The effects of reflected waves from the far end are not seen yet as the domain length is much longer. Any reflections from the left boundary are also invisible. For $\omega^{*}=0.5$, significant differences in the force patterns are also seen for different material characteristics. When the elastic constant $E$ is reduced from $200 \mathrm{GPa}$ to $0.8 \mathrm{GPa}$, the force amplitude hardly increases (cases (f) and (g)). However, further reduction of the constant to $0.2 \mathrm{GPa}$ shows a marginal increase in the force magnitude. However, a significant (about $40 \%$ ) increase in transient force amplitudes is seen when the elastic constant is further reduced to $0.1 \mathrm{GPa}$. This once again, confirms a significant need for transient fluid-structure coupling. This means that as the frequency reduces, the increase of force magnitudes based on fluid-structure interaction also reduces. This is expected as waves have longer lengths when the frequency reduces. As the amplitude of paddle operation is kept constant, waves will be flatter for $\omega^{*}=0.5$ and hence forces should be less. However, we feel 


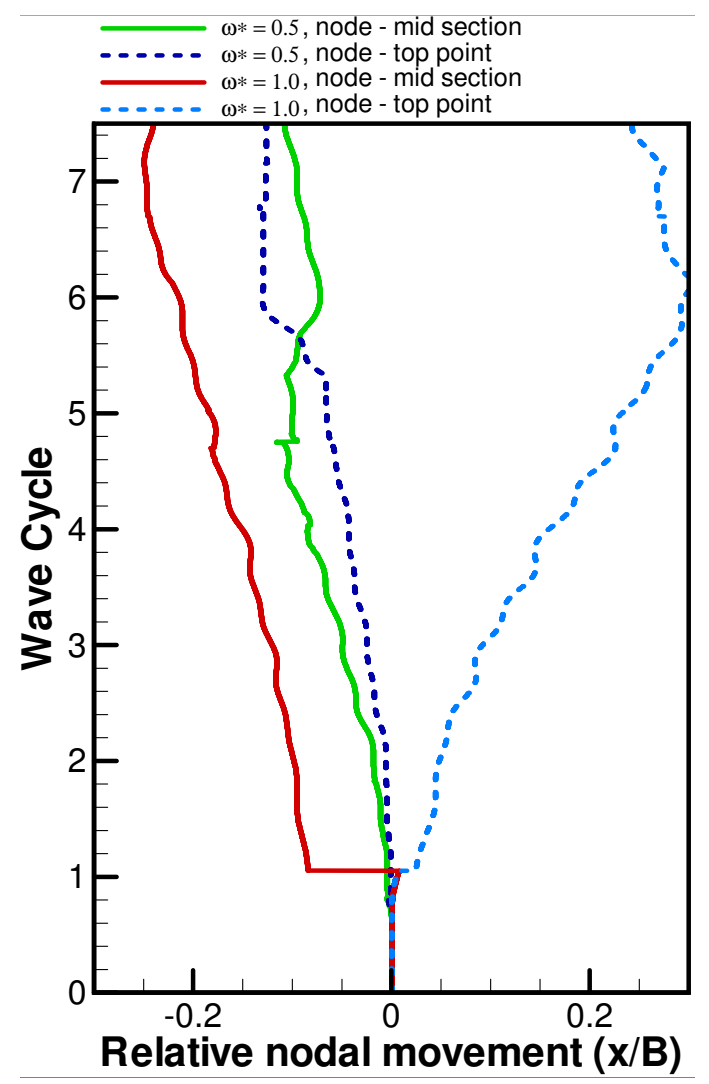

Figure 2: Time history of specific nodes for a structure with $E=0.1 \mathrm{GPa}$.

that for the particular structural configuration considered here, formation of vortices around the top of the structure may have some effect on the transient dynamics and hence the forces. Therefore, these forces should be treated as indicative values, merely demonstrating the nonlinear coupled behaviour.

Further understanding of the FSI process demanded analysis of other physical quantities. One of the parameters could be time history of typical nodes on the structure. For this purpose, the time histories of the two nodes located at mid-height and at full-height of the structure for an elas- 
tic constant $0.1 \mathrm{GPa}$ are considered as this was the structure that showed maximum nonlinearity (Figure 1). Figure 2 shows time histories for two different frequencies $\left(\omega^{*}=0.5\right.$ and $\left.\omega^{*}=1.0\right)$ against the relative structural motions $x / B$, where $B$ is the width of the structure. Comparison of nodal histories shows a strong frequency dependence of the structural motion, an expected tendency in dynamic systems. For $\omega^{*}=0.5$, the whole structure seems to move in phase. Whereas, for $\omega^{*}=1.0$, the top node and mid node move out of phase. This is reasonable as the $\omega^{*}=0.5$ wave is much longer in period. Hence, all the nodes in the structure behave in unison as if they are facing a slowly varying current. A minor high frequency mode could be observed superposed with the main wave mode. This secondary mode could correspond to the natural frequencies of the structure. In summary, a strong correlation between the wave and structural frequencies is captured in the time domain nonlinear FSI approach, and the structural behaviour is totally time dependent showing traces of its natural frequencies over the primary motions.

Figure 3 shows an energy spectral plot of the free surface for $\omega^{*}=1.0$ with the simulations conducted for structures of two different materials. This frequency is chosen as it showed the maximum displacement and dynamic force nonlinearities. A spectral analysis of the free surface profile is carried out in order to identify frequencies other than the incident wave frequency generated by the structural interaction. Comparing the transient force pattern in Figure 1 for $\omega^{*}=1.0$, it was expected that the free surface profile would show a trace of other high frequency disturbances created by the structural motions. The spectral densities for the two materials are plotted in the wave number space as the free surface profile is a spatial quantity. See that the spectral densities for the two materials follow each other throughout wave number space. They show a peak at $k \approx 1.2$, corresponding to the incident wavelength of about $5.2 \mathrm{~m}$. There is a second frequency seen at $k \approx 4.8$. This may correspond to the secondary cycle seen in the nodal displacements. However, this needs further confirmation with more simulations and experiments. 


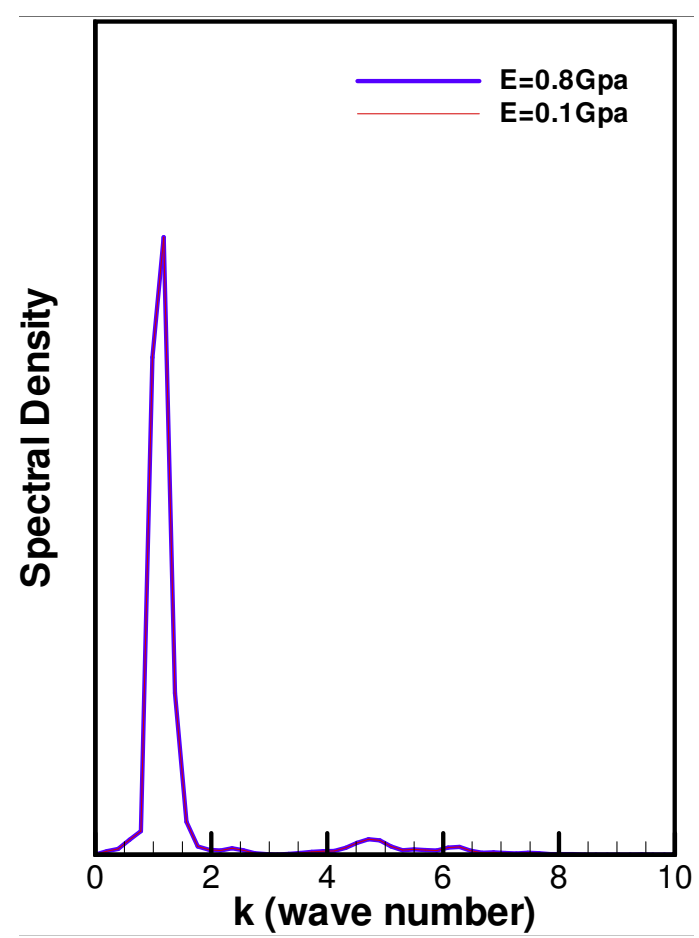

Figure 3: Spectra energy plot of free surface in the $k$-space.

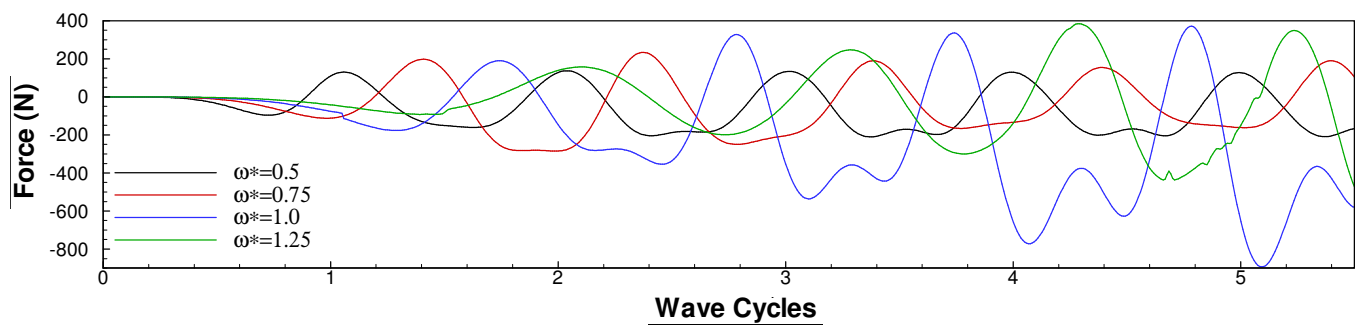

FigURE 4: Effect of incident wave frequencies on the dynamic force. 
To clearly establish a link between magnification of dynamic FSI effects in relation to the wave frequency, transient forces are simulated for $\omega^{*}=0.5$, $0.75,1.0$ and 1.25 for a particular structure. The dynamic force histories are plotted in Figure 4 for all these frequencies. This exercise is performed for $E=0.1 \mathrm{GPa}$ to achieve maximum FSI effects. The plots once again reveal that there is an increase in the dynamic forces as the frequency increases up to $\omega^{*}=1.0$. Further increase in the frequency does not result in an increase in the dynamic forces.

In all these exercises, a strong coupling between wave frequency, structural displacements and dynamic forces are noticed. However, sufficient data, be it experimental or numerical, is not available to pinpoint specific correlation between them. We are currently considering a more accurate fluiddynamic solver, different material and structural models, and advanced solver and meshing techniques. All these are challenges for further studies.

\section{Concluding remarks}

The use of a nonlinear velocity potential and linear elasticity theory is demonstrated in studying fluid structure interaction. This was accomplished by using an unstructured meshing technique in both the fluid and structural solvers. This has helped in progressing our effort to create a Numerical Wave Tank with FSI capabilities.

Various test conditions were simulated and analysed. In summary, the force applied on the structure changes due to the structure's elastic response, in both the elastic deformation and the sway. The dynamics of the structure has a nonlinear effect on transient hydrodynamic pressure forces. In addition, the transient forces have a strong correlation with material properties of the structure. The increase in transient force amplitudes for various elastic constants also varies as the wave frequency is changed. In general, as the wave frequency reduces, the gap between elastic and rigid structure dynamics also 
reduces. Hence, the fluid-structure coupling is more significant at high frequencies. Analysis of the structural dynamics, resulting free-surface profiles and dynamic forces establishes the existence of critical frequency. However, its relationship with the structural properties is still to be established. Future studies should focus on the relationship between the importance of material characteristics and wave frequencies as for as dynamic interactions are concerned. Also, the effect of fluid-structure coupling on the free surface profiles should be analysed over a wide range of frequencies. Sophisticated algorithms in the area of mesh handling and radiation dampers should be employed for this purpose.

\section{References}

[1] Murali, K., Performance Analysis of a Cage Floating Breakwater. Ph.D Thesis, Indian Institute of Technology, Madras, India, 1996. C1364

[2] Wu, G. X. and Taylor, R. E. Finite element analysis of two-dimensional non-linear transient water waves. Applied Ocean Research, 16, 363-372, 1994. C1357

[3] Wu, G. X. and Taylor, R. E. Time stepping solutions of the two-dimensional non-linear wave radiation problem. Ocean Engineering, 22:(8), 785-795, 1995. C1357

[4] Bathe, K. J., Finite Element Procedures. Prentice - Hall, Englewood Cliffs, N. J., USA, 1996. C1358

[5] Farhat, C. and Lesoinne, M. Higher-order Staggered and Subiteration Free Algorithms for Coupled Dynamic Aeroelasticity Problems. 36th Aerospace Sciences Meeting at Exhibit 5, AIAA 98-0516, Reno NV., 1998. C1359 
[6] Murali, K, Jing Lou, and Kurichi Kumar. An Unstructured model Simulations for Singapore Strait. Maritime and Port Journal, Singapore, 117-127, 2002. C1360

[7] Sudharsan, N. M., Ajaykumar, R., Murali, K. and Kurichi, Kumar. A comparative study of dynamic mesh updating methods used in the simulation of fluid-structure interaction problems with non-linear free-surface. Journal of Mechanical Engineering Science, Proceedings of the Institution of Mechanical Engineers U.K., Part C, 218:3, 283-300, 2004. C1360

[8] Sudharsan, N. M., Murali, K. and Kurichi Kumar. Preliminary investigations on non-linear fluid-structure interaction of an offshore structure. Journal of Mechanical Engineering Science, Proceedings of the Institution of Mechanical Engineers U.K., Part C, 217, 759-765, 2003. C1360

[9] Pal, N. C., Sinha, P. K. and Bhattacharyya, S. K. Finite element coupled slosh analysis of a rectangular liquid filled laminated composite tanks. Journal of Reinforced Plastics and Composites, 18:15, 1375-1407, 1999. C1362 